\title{
Synthesis of the force and displacement methods of structural mechanics. The planar truss case
}

\author{
Krzysztof Hetmański ${ }^{1, *}$ \\ ${ }^{1}$ Faculty of Civil Engineering, Warsaw University of Technology, 16 Armii Ludowej St., Warsaw 00- \\ 637, Poland
}

\begin{abstract}
The papers delivers a new computational method of statical analysis of frames, well suited for both the 2D and 3D settings and easily generalizable to the case of the presence of big axial forces. The method is based on the compatibility equations which have their roots in the force method, while the unknowns refer to the displacement method. These equations involve also the unknown cross sectional forces which are the force method unknowns. The method proposed can be viewed as the displacement method with an implicit procedure of deriving the stiffness equations.
\end{abstract}

\section{Introduction}

In contrast to the elasticity theory in which the stress-based and displacement-based approaches are complementary, the force method (FM) and the displacement method (DM) are usually treated independently, even in separate chapters, cf. the literature cited [1-6]. The aim of the present paper is to put forward a synthesis of the FM and DM methods by focusing attention on the statics of planar trusses. Yet the ideas behind refer to all skeletal structures, including 3D case. The main assumption is the linear elasticity setting. The load is assumed in the form such that the truss bars are not subject to bending. The transverse load is allowed only in specific cases.

\section{The approach free from the matrix algebra formalism}

Independently of the approach applied the first step is determination of the unknown kinematic data (or the degrees of freedom) as independent and non-zero nodal displacements, cf. Fig.1.

\footnotetext{
*Corresponding author: k.hetmanski@plusnet.pl
} 


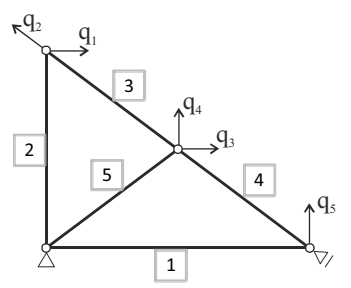

Fig. 1. An exemplary choice of the degrees of freedom of a given planar truss.

The degrees of freedom are collected in a column vector as follows

$$
\mathbf{q}=\left[q_{1}, \cdots, q_{s}\right]^{T},
$$

where s represents the number of degrees of freedom. At each bar we determine one node as a first node (the index of this starting node is : "i") and the other node (of index "k") as the end node (or second node). At the node "i" we release the constraint associated with the axial force and replace this constraint by the pair of mutual reactions, see Fig.2. The left axial force is denoted by $N_{i}^{e}$. Let us note that the axial force can vary along the bar axis if the axial external load is applied along the bar. Then the axial force is not equal to $N_{i}^{e}$. The resultant of the external axial load is denoted by $Q_{w}^{e}$, see Fig.2. This resultant force is attached to the node " $\mathrm{k}$ ".

The number of truss members equals $\mathrm{p}$.

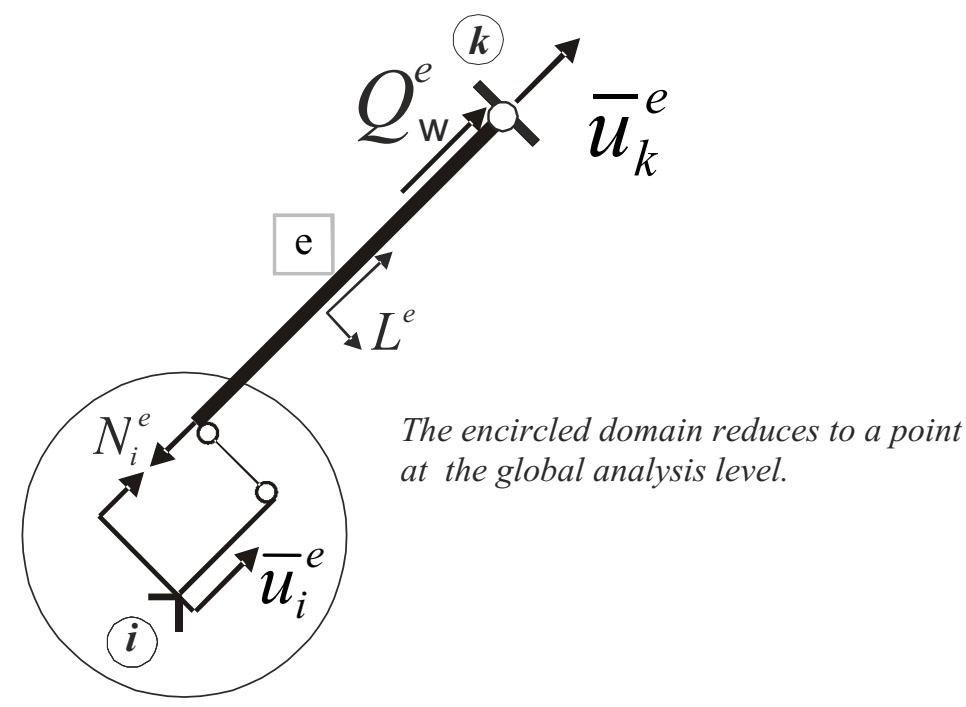

Fig. 2. Releasing the constraint associated with the axial force at point $i$.

The process of releasing the constraints transforms the truss into a mechanism for which the virtual displacements can be subsequently imposed, see Eq.(2) below. Let the virtual displacements be taken in the form of the following linearly independent column vectors.

$$
\overline{\mathbf{q}}_{j}=\left[0, \cdots, \bar{q}_{j}=1, \cdots, 0\right]^{T}, j=1, \cdots, s .
$$


Thus, the number of virtual states is equal to the number of degrees of freedom. For each of these states we associate one equilibrium equation which follows from the virtual work equation. The latter reads

$$
-\sum_{e} N_{i}^{e} \underbrace{\left(\bar{u}_{j k}^{e}-\bar{u}_{j i}^{e}\right)}_{\bar{\Delta}_{j}^{e}}+\bar{L}_{j}=0, j=1, \cdots, s .
$$

where $\bar{u}_{j i}^{e}, \bar{u}_{j k}^{e}$ are nodal virtual displacements projected onto the bar direction e while $\bar{L}_{j}$ is the virtual work of forces applied to the nodes and of the forces $Q_{w}^{e}$ reduced to the nodes. If, exceptionally, there are applied transverse loads, then the load should be replaced by the statically equivalent point loads applied to the nodes. Then these forces will contribute to the expression for the virtual load $\bar{L}_{j}$. Let us introduce the quantity $\bar{\Delta}_{j}^{e}$ which expresses the change of the distance between the nodes $\mathrm{i}$ and $\mathrm{k}$ measured along the bar axis, caused by the virtual displacements $\overline{\mathbf{q}}_{j}$. By the method of elimination of the pivotal element from the equilibrium equations (3) one can eliminate $\mathrm{s}$ forces $N_{i}^{e}$, provided that the subsequent equations are solvable. If undetermined or mutually contradictory equations appear, this would mean that the truss is geometrically instable. This case is excluded from our discussion. The member forces which remain upon the mentioned elimination process may be treated as redundants of the force method and their appearance indicates that the basic structure is correctly constructed. On the other hand, if all the member forces can be computed, the initial structure is statically determinate. Hence we conclude that the degree of statical indeterminacy equals the difference between the number of cross sectional forces of the truss bars and the number of degrees of freedom of its nodes. One can note that this statement holds for all other types of skeletal structures. The next key step is to construct the compatibility equations referring to the released constraints, where the gap displacements $\delta_{i}^{e}$ appear, cf Fig. $3 . \quad \delta_{i}^{e}$ measures the distance between the sections being cut.

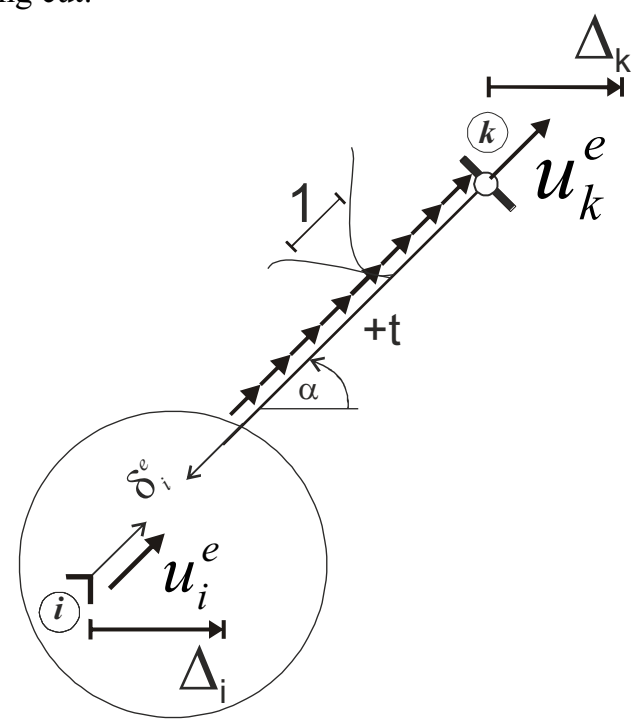

Fig. 3. Relative displacement at point $i$. 
The same figure shows selected span loads and the kinematic loads referring to the nodes of the given bar. This set of loads affects the unknown displacement in the term $\delta_{i 0}^{e}$. The exemplary formula is given below

$$
\delta_{i 0}^{e}=\left\{\begin{array}{l}
\alpha_{t} t L^{e} \\
\Delta_{i} \cos \alpha-\Delta_{k} \cos \alpha \\
1
\end{array}\right.
$$

The unit error of assembling of the members makes it possible to determine the influence line of the axial force of the truss. Thus the compatibility equations read

$$
\delta_{i 0}^{e}+N_{i}^{e} \frac{L^{e}}{(E A)^{e}}+\underbrace{u_{i}^{e}-u_{k}^{e}}_{-\Delta^{e}}=0, e=1, \cdots, p,
$$

Let us note that Eqs.(5) still hold if the bars are inextensible. The expression $\Delta^{e}=u_{k}^{e}-u_{i}^{e}$ should be expressed by the components of vector, by projecting the displacements of nodes on the directions of the axes of the connecting bars. This can be concisely written in the matrix form in the next section. Now let us mention only that these relations are linear with respect to the components of $\mathbf{q}$. Thus, in equations (5) there appear the bar forces $N_{i}^{e}$ not eliminated from (3) as well as all components of $\mathbf{q}$. Then, by a simple elimination one can compute these unknowns, which ends the analysis. Let us check the balance of unknowns and equations. The unknowns are the bar forces for which we have the same number of compatibility equations. On the other hand we have one equilibrium equation for each displacement, which proves that the balance holds. The presented algorithm is not to support the done- by -hand computations, but it was efficiently used in the program code, extended also to the bending frames in 2D.

Let us discuss now the matrix form of (3), (5). The matrix equations are attractive, yet a direct implementation is non effective. A lot of zero components appear which makes the computations ineffective. The problem can be solved by using some refined algorithms, but then the conciseness of the matrix equations is lost.

\section{The matrix formulation}

The geometric equations linking the bars elongations (6) with the nodal displacements are crucial here. They read

$$
\boldsymbol{\Delta}=\left[\begin{array}{c}
\Delta^{1} \\
\vdots \\
\Delta^{p}
\end{array}\right]_{p x 1}=\left[\begin{array}{c}
u_{k}^{1}-u_{i}^{1} \\
\vdots \\
u_{k}^{p}-u_{i}^{p}
\end{array}\right]=\mathbf{B} \cdot \mathbf{q}
$$

where $\mathbf{B}$ is the rectangular matrix of dimensions $\mathrm{p}$ by s. Let us introduce the column vectors as follows 


$$
\mathbf{N}=\left[\begin{array}{c}
N_{i}^{1} \\
\vdots \\
N_{i}^{p}
\end{array}\right], \overline{\mathbf{L}}_{z}=\left[\begin{array}{c}
\bar{L}_{1} \\
\vdots \\
\bar{L}_{s}
\end{array}\right],
$$

The equilibrium equations assume the concise form below

$$
\mathbf{B}^{T} \mathbf{N}=\overline{\mathbf{L}}_{z}
$$

Upon multiplying (5) $\frac{(E A)^{e}}{L^{e}}$ by one finds

$$
N_{i}^{e}=\frac{(E A)^{e}}{L^{e}} \Delta^{e}-\frac{(E A)^{e}}{L^{e}} \delta_{i 0}^{e}, e=1, \cdots, p
$$

Let us introduce the matrices

$$
\mathbf{E}=\left[\begin{array}{ccc}
\frac{(E A)^{1}}{L^{1}} & \cdots & 0 \\
\vdots & \ddots & \vdots \\
0 & \cdots & \frac{(E A)^{p}}{L^{p}}
\end{array}\right]_{p x p}, \mathscr{D}_{\mathbf{o}}=\left[\begin{array}{c}
\delta_{i 0}^{1} \\
\vdots \\
\delta_{i 0}^{p}
\end{array}\right]_{p x 1}
$$

By using (6) we rearrange the above formulae to the form

$$
\mathbf{N}=\mathbf{E B q}-\mathbf{E} \mathscr{\theta}_{\mathbf{0}}
$$

Substitution of (11) into (8) leads to the governing equation in which the displacements are the main unknowns

$$
\begin{aligned}
\underbrace{\mathbf{B}^{T} \mathbf{E B} \mathbf{q}}_{\mathbf{K}} & =\underbrace{\overline{\mathbf{L}}_{z}+\mathbf{B}^{T} \mathbf{E} \mathscr{D}_{\mathbf{0}}}_{\mathbf{Q}}, \\
\mathbf{K q} & =\mathbf{Q} \Rightarrow \mathbf{q}=\mathbf{K}^{-1} \mathbf{Q} .
\end{aligned}
$$

Coming back to (11) we compute the forces in bars.

\section{Extension to other types of skeletal structures}

Let us consider planar frames. The displacements adjacent to a node are now introduced as in Fig.4. We note that the independent angles of rotation appear. 


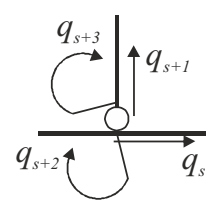

Fig. 4. Degrees of freedom in frame node.

We assume that the bars of frames have arbitrary shape and are clamped at two different nodes of the structure. Thus releasing the constraints around the node $i$ requires introduction of pairs of cross sectional forces, see Fig.5. Here L stands for the length of the chord of the bar.

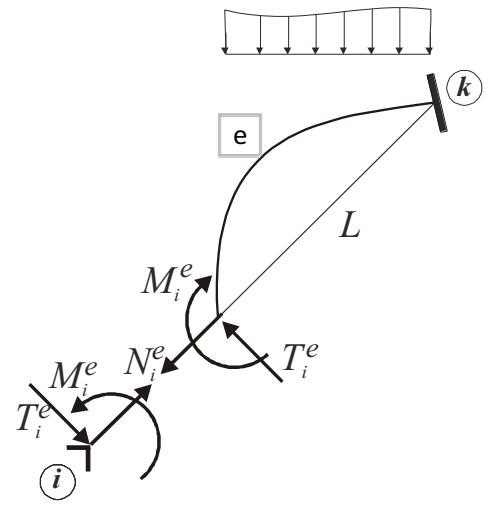

Fig. 5. Releasing the constraints at a node in the direction of one bar.

By imposing virtual displacements according to (2) and applying the virtual work equation one finds the equations of equilibrium and its number is equal to the number of unknown degrees of freedom of the frame. On the other hand the compatibility equations are cleared up in Fig.6.

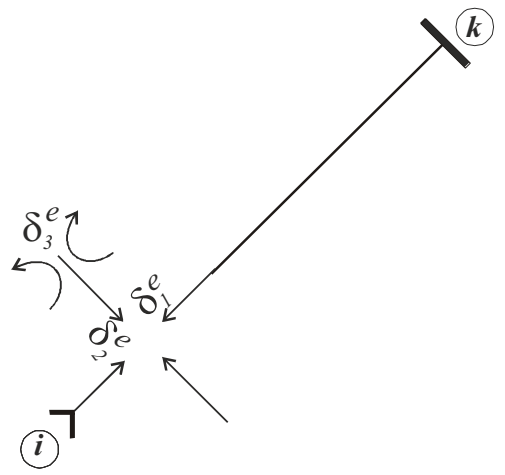

Fig. 6. Compatibility equations.

The compatibility equations are expressed by

$$
\begin{aligned}
& \delta_{1}^{e}=\overbrace{N_{i}^{e} \delta_{11}^{e}+T_{i}^{e} \delta_{12}^{e}+M_{i}^{e} \delta_{13}^{e}+\delta_{10}^{e}}^{\text {the terms corresponding tothe loads applied tothe element: }} \quad \overbrace{+u_{i} \cos \alpha+v_{i} \sin \alpha-u_{k} \cos \alpha-v_{k} \sin \alpha}^{\text {the terms determined by nodal displacements: }}=0 \\
& \delta_{2}^{e}=N_{i}^{e} \delta_{21}^{e}+T_{i}^{e} \delta_{22}^{e}+M_{i}^{e} \delta_{23}^{e}+\delta_{20}^{e} \\
& \delta_{3}^{e}=\quad N_{i}^{e} \delta_{31}^{e}+T_{i}^{e} \delta_{32}^{e}+M_{i}^{e} \delta_{33}^{e}+\delta_{30}^{e}
\end{aligned}
$$


The physical meaning of the selected coefficients is cleared up in Fig.7.

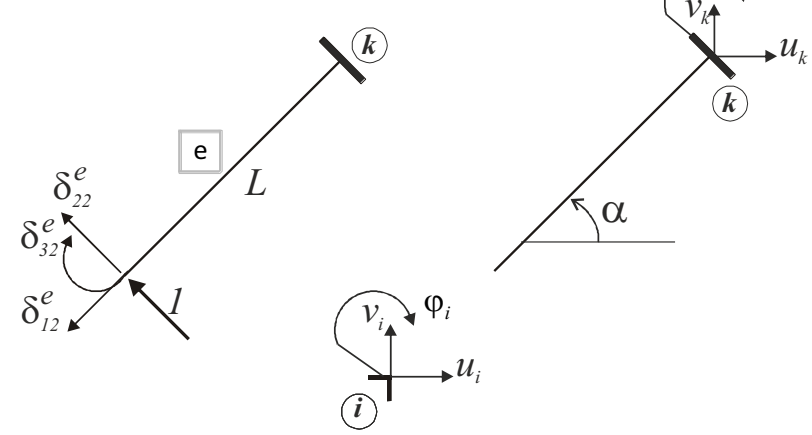

Fig. 7. Selected coefficients in Eqs.(13)

As already mentioned the computer program has not been constructed upon the matrix equations.

The method can be extended to the case of frames subject to big axial forces and to 3D frames in general.

\section{Concluding remarks}

The compatibility equations (5), (13) deliver a synthesis of the FM and DM methods. The compatibility equations have their roots in the FM while the unknowns involved refer to the DM method. These equations involve also the unknown cross sectional forces which usually are FM unknowns. One can say that the method shown is the DM method with an implicit procedure of deriving the stiffness equations. The two approaches discussed reveal a certain paradox. The basis of the computer program is an algorithm which does not refer to the matrix equations formalism.

\section{References}

1. W. Nowacki, Mechanika Budowli, t. I, II (PWN, Warszawa, 1965)

2. C. Branicki, R. Ciesielski, Z. Kacprzyk, J. Kawecki, Z. Kaczkowski, G. Rakowski, Mechanika Budowli. Ujęcie Komputerowe (Arkady, Warszawa, 1991)

3. A. Gawęcki, Mechanika materiałów i konstrukcji prętowych Tom I i II (Wydawnictwo Politechniki Poznańskiej,1998)

4. T. Lewiński, J.Theoret.Appl.Mech. 2(39), 307-322 (2001)

5. G. Dzierżanowski, W. Gilewski, K. Hetmański, T. Lewiński, Zbiór zadań z mechaniki konstrukcji prętowych. Zagadnienia Statyczne (Oficyna Wydawnicza PW, 2014) 